\title{
Using Ultrasound to Teach Female Reproductive Physiology
}

\author{
${ }^{1}$ Herbert F Janssen, ${ }^{2}$ Sanja Plavsic Kupesic
}

${ }^{1}$ Professor of Physiology, Department of Medical Education and Department of Orthopedic Surgery and Rehabilitation, Paul L Foster School of Medicine, Texas Tech University Health Sciences Center, 5001 El Paso Drive, El Paso, Texas 79905, USA

${ }^{2}$ Professor and Clinical Professor of Obstetrics and Gynecology and Radiology, Department of Medical Education, Paul L Foster School of Medicine, Texas Tech University Health Sciences Center, 5001 El Paso Drive, El Paso, Texas 79905, USA

Correspondence: Herbert F Janssen, Professor of Physiology, Department of Medical Education and Department of Orthopedic Surgery and Rehabilitation, Paul L Foster School of Medicine, Texas Tech University Health Sciences Center, 5001 El Paso Drive, El Paso, Texas 79905, USA e-mail: herb.janssen@ttuhsc.edu

\begin{abstract}
Physiology ties together many related disciplines including anatomy, histology, biochemistry, and cell biology. Mastering the concepts of physiology is essential to understanding the principles of medicine. Learning physiology requires acquisition of facts, but this alone provides little in the way of useful knowledge. Currently, educators are challenged with the task of presenting physiology in a manner that encourages students to actively learn the required material. Didactic lectures were considered antiquated a century ago by Abraham Flexner when he reported on the status of medical education in the United States and Canada. Unfortunately, his comments did little to dissuade the use of lecture as a primary teaching method in medical education. Today we have the opportunity to develop new methods to present material in a manner that encourages active learning and understanding of concepts. Ultrasound imaging is a tool useful in presenting many organ systems in physiology. This is especially true of the female reproductive system. Ultrasound equipment can be used to develop still images of the ovaries, fallopian tubes, uterus, and the developing fetus. It can also provide video clips showing the reproductive organs in juxtaposition with the surrounding tissue or images of the fetus complete with heart sounds and vascular flow. During more advanced training, the students can develop hands-on skills using the ultrasound to identify and evaluate structures.
\end{abstract}

Keywords: Ultrasound, teaching, physiology, female reproduction, ovaries, uterus, fetus.

\section{INTRODUCTION}

There is a difference between teaching and learning. There is also a difference between facts and concepts. Teaching facts using didactic lectures is of little use. Material taught in this manner is usually not learned and even if facts are memorized, the student has accomplished little if they do not understand the underlying concepts. If a student learns how to use information to solve a problem and gains an appreciation of the underlying concepts, the information can be put to practical use.

In 1910, Abraham Flexner's now famous report on medical education in the United States and Canada spoke against didactic lecturing. In this report he stated:

"In methods of instruction there is, once more, nothing to distinguish medical from other sciences. Out-and-out didactic treatment is hopelessly antiquated; it belongs to an age of accepted dogma or supposedly complete information, where the professor 'knew' and the students 'learned.' The lecture indeed continues of limited use. It may be employed in beginning a subject to orient the student, to indicate relations, to forecast a line of study in its practical bearings; from time to time, too, a lecture may profitably sum up, interpret, and relate results experimentally ascertained". ${ }^{1}$

Flexner is now criticized by some for having promoted the use of deductive reasoning as a means of problem solving in medicine education; however, even those who are most critical generally accept his assertion that didactic lecture is of little value. It also seems that critics often forget the miserable state of medical education Flexner encountered in the early 20th century.

Unfortunately, Flexner's statement regarding the antiquated nature of the lecture technique did little to alter medical pedagogy over the last century. The age-old lecture approach has been passed down from professor to student and is again repeated. Writing tablets have been replaced by computers and chalk boards by PowerPoint, but the basic underlying principle remains the same. The information passes from the notes of the professor to the notes of the student without being processed by the brain of either. The professor teaches but the student does not learn. Active 
thinking is discouraged or prohibited in the classroom due to the limited time allotted for each professor to teach a list of facts. If learning is accomplished, it must be done by the student outside of classroom. In too many cases professors takes credit for the students who succeed and write off the others as failures, claiming they are incapable of mastering the required material.

\section{TEACHING REPRODUCTIVE PHYSIOLOGY USING ULTRASOUND}

Physiology is the study of function. It ties together the anatomy, histology, biochemistry, and cell biology of a living organism. It forms the underpinnings on which the practice of medicine is based. Using simple lecture without accompanying laboratories, demonstrations and/or handson learning approaches to teach this dynamic subject is a poor choice.

Physiologists should explore new methods of presenting topics that capitalize on advances in technology and instrumentation. Such approaches provide students with an opportunity to appreciate the intricacies of physiology and engage in active learning. Modern ultrasound equipment provides one such option and can be used to enhance the presentation of many organ systems. When used to explore the female reproductive system, ultrasound provides a useful tool to examine both the functional anatomy of the organs and the development of a growing fetus. It can bring to life the factual material found in textbooks and give added meaning to structures observed during anatomical dissections. Still images captured with ultrasound give meaning to illustrations or photographs found in textbooks and anatomical atlases. Ultrasound images captured on video allow students to visualize structures in a dynamic relationship with surrounding tissue. Additionally, videos of fetal movement at different stages of development can be presented and analyzed. Live demonstrations give the student the opportunity to more completely appreciate the nature of an ultrasound examination; however, videos and still images can be displayed later and remain valuable teaching tools. When ultrasound images of the structures are coupled with video showing the operator, patient, set up procedure, and appropriate sonographic techniques, distance education can provide learning opportunities similar to the live demonstration. This also opens the door for the development of educational material provided through online courses accessible at the student's request.

In the hands of the trained professor, ultrasound can be used to demonstrate both normal and pathological conditions. It can also be used to illustrate the development of the embryo starting only days after implantation. This is particularly true for the more recently introduced 3-D and 4-D units that provide lifelike images in real time.

In more advanced training, the student can gain valuable hands-on experience with the ultrasound equipment while learning to perform a patient evaluation. Many phantoms exist that can be used to provide the novice student with needed experience. The noninvasive nature of ultrasound also allows repeated use of the device without fear of injuring either the subject or operator. When a student has the opportunity to use ultrasound equipment under the tutelage of a physician educator, they can gain experience in the art and science of patient evaluation, while adding to their understanding of structure and function of the reproductive organs.

Unlike many devices, ultrasound provides immediate feedback. In the case of the pregnant patient, this can be a powerful experience. It gives a visual and audible reality to the living being that is developing within the mother. For her, this is an emotional experience that confirms the presence of the child she is carrying. For the physician, the images provide an objective method that can be used to evaluate the development of the fetus and its progression toward maturity.

\section{ULTRASOUND IMAGES USEFUL IN TEACHING}

The following is a collection of images of the female reproductive system obtained with various ultrasound devices (Figs 1 to 10). Obviously, other images and/or videos can be obtained as needed to present the various topics. These provide a powerful tool that teaches physiology while reinforcing other basic science disciplines.

\section{REFERENCE}

1. Flexner A. Medical education in the United Stated and Canada. Bulletin number four. The Carnegie Foundation: New York, 1990. 


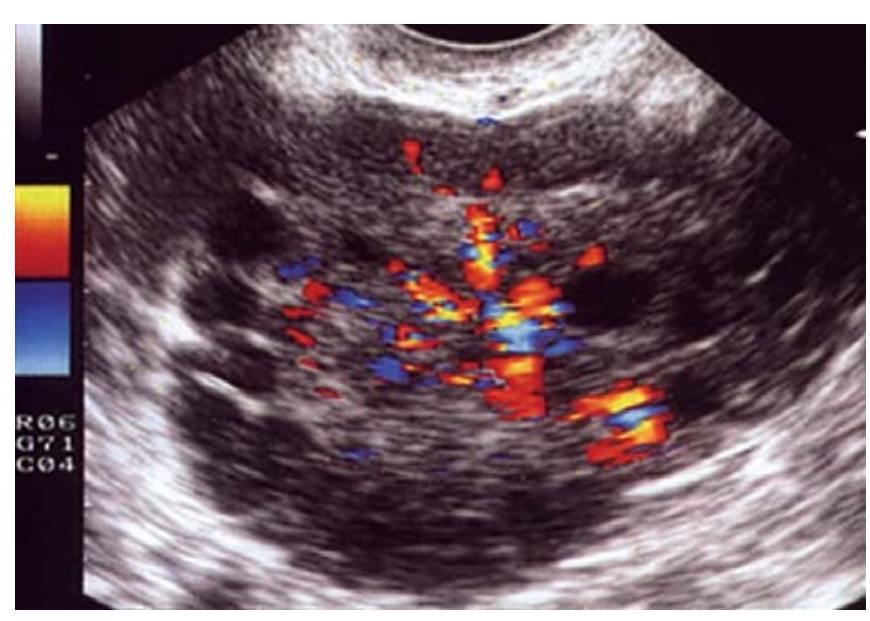

Fig. 1: Polycystic ovary in an infertile patient with oligomenorrhea, acne and hirsuitism. Color Doppler reveals increased intraovarian vascularity

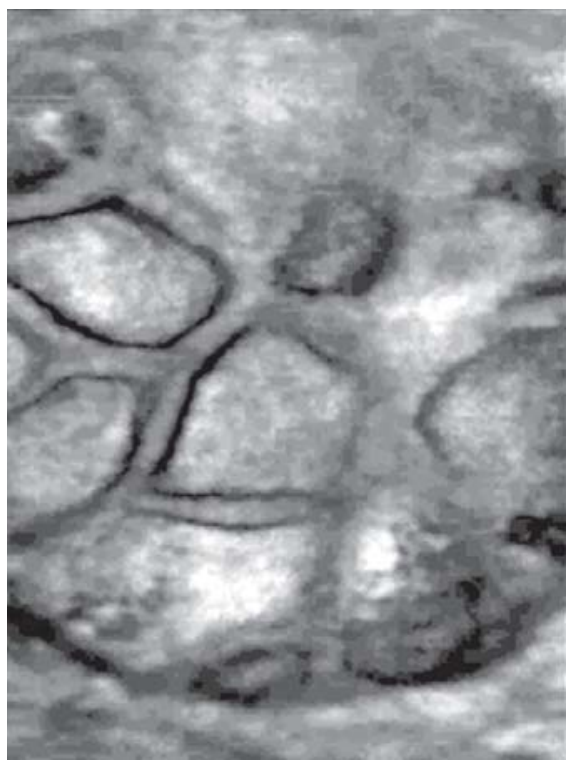

Fig. 3: The same patient. Three-dimensional ultrasound of ovarian hyperstimulation in a patient undergoing ovarian ovulation induction

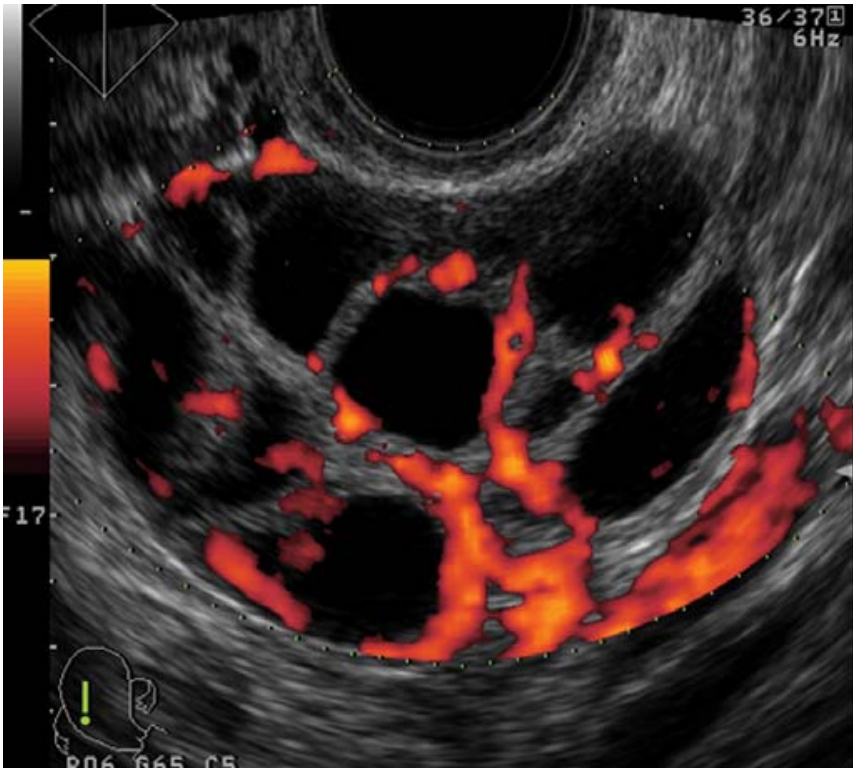

Fig. 2: Power Doppler ultrasound of ovarian hyperstimulation in a patient undergoing ovarian ovulation induction. Dilated vessels at the periphery of the developing follicles are clearly visualized

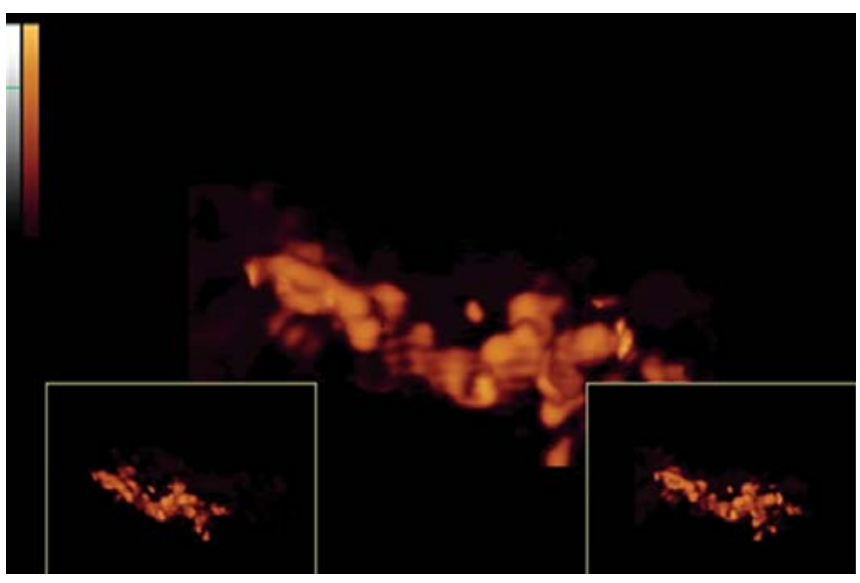

Fig. 4: Patent fallopian tube demonstrated by three-dimensional power Doppler ultrasound

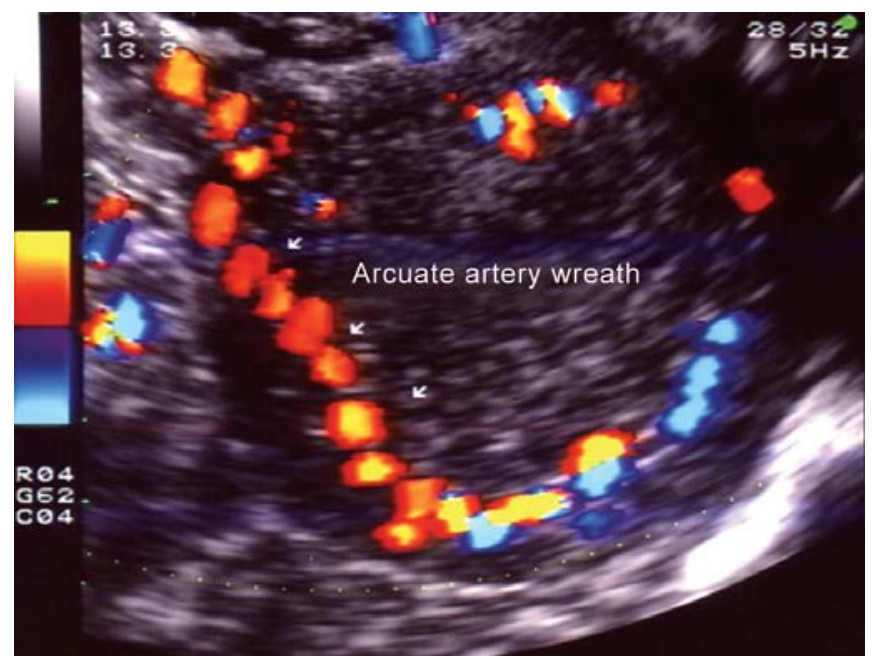

Fig. 5: Transvaginal color Doppler ultrasound of the uterine circulation 


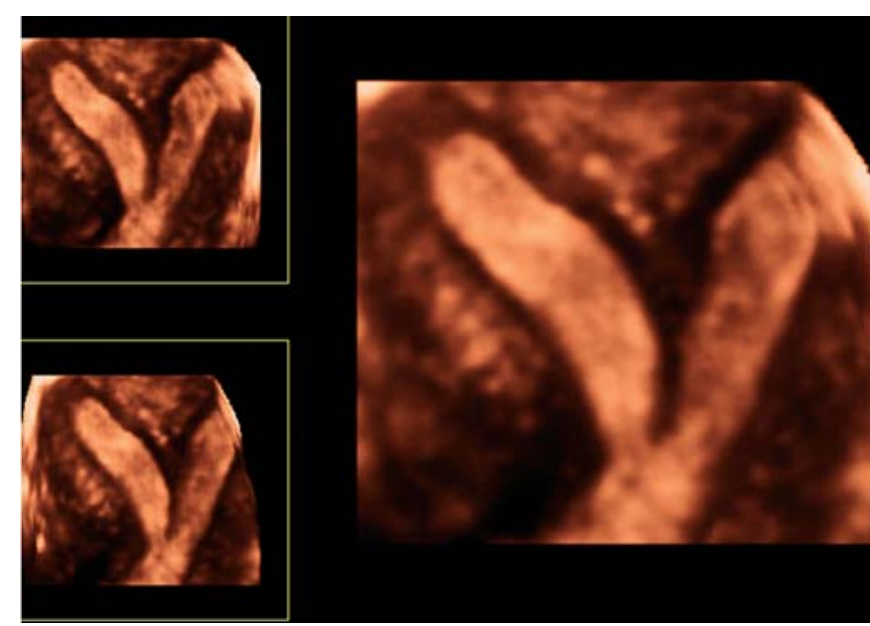

Fig. 6: Three-dimensional ultrasound and frontal reformatted section of a septate uterus. Note clear division of the uterine cavity in the upper half of the uterine cavity

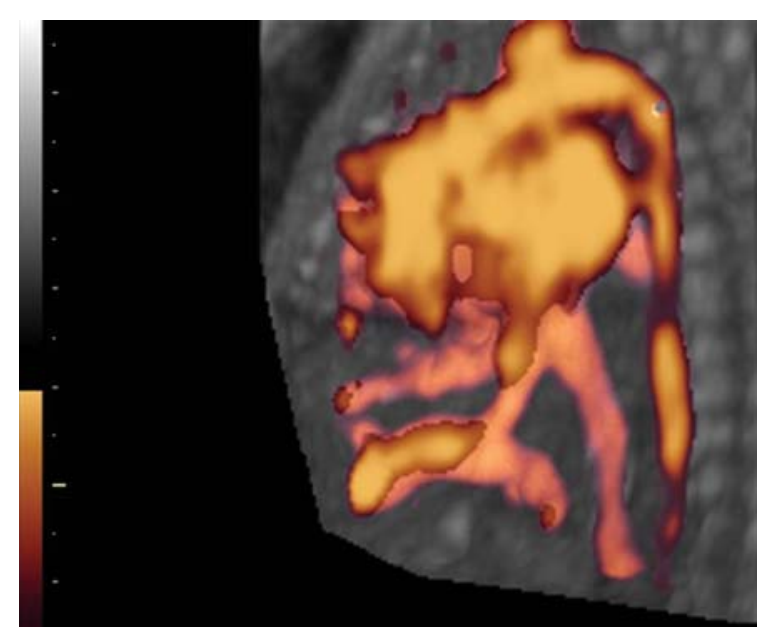

Fig. 8: Fetal circulation assessed by three-dimensional power Doppler ultrasound. (Embryology Image Library Editors: Sanja Plavsic Kupesic, $\mathrm{MD}, \mathrm{PhD}$ and Guillermo Azumendi, MD)

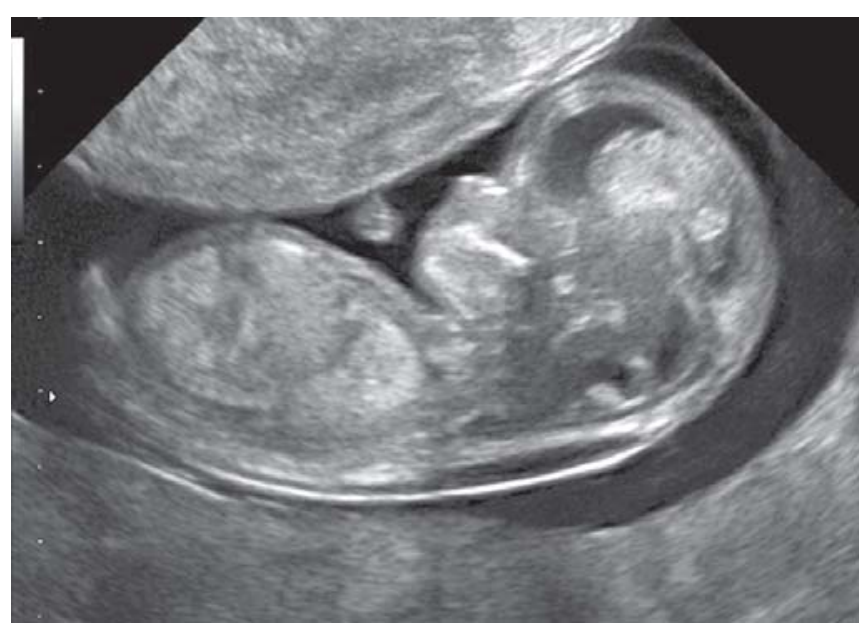

Fig. 7: Transvaginal ultrasound of the fetus at 12 weeks gestation. Nuchal translucency is clearly visualized

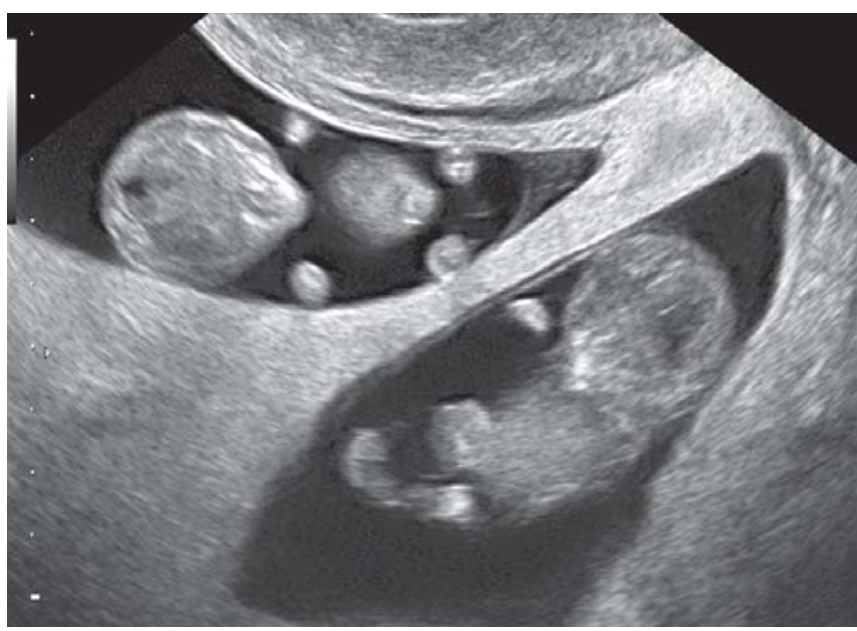

Fig. 9: Two-dimensional ultrasound scan of dichorionic diamniotic twin pregnancy at 10 weeks' gestation

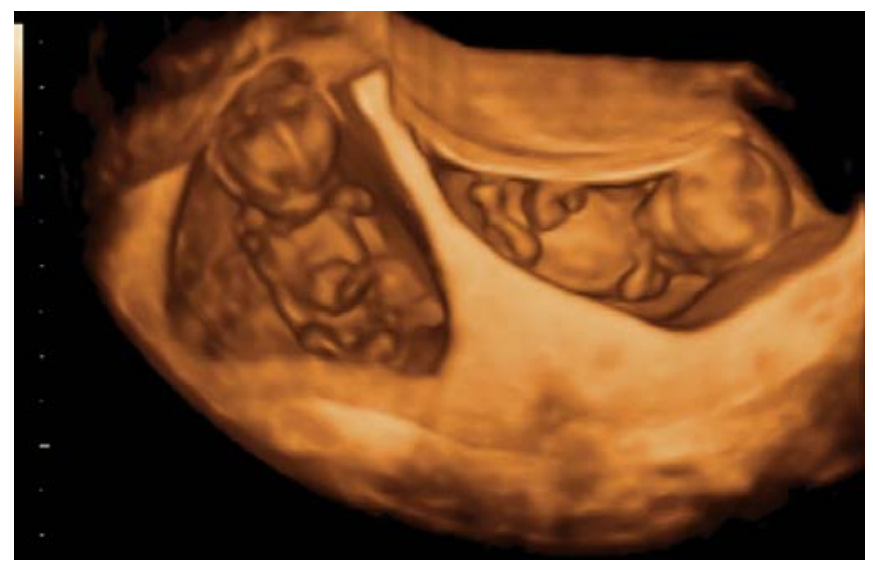

Fig. 10: Three-dimensional ultrasound of dichorionic diamniotic twin pregnancy at 10 weeks' gestation 